\title{
Alternating Current Polishing and Electroetching of Metals ${ }^{*}$
}

by

Kaye Allan Johnson

This paper was submitted for publication in the open literature at least $P_{\text {months }}$ prior to the issuance date of this Microcard. Since the U.S.A.E.C. has no evidence that it has been published, the paper is being distributed in Microcard form as a preprint.

\author{
University of California \\ Los Alamos Sclentific Laboratory \\ Los Alamos, New Mexico
}

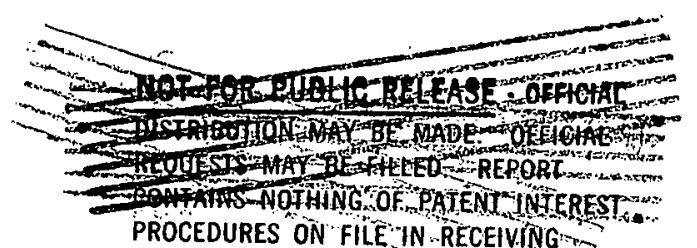

PROCEDURES ON FLLE'N RECEVING

SECTION.

1

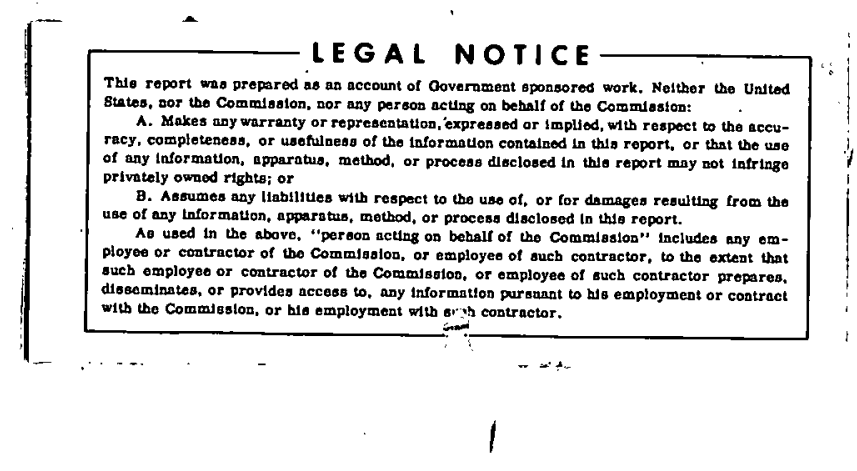

* Work performed under the auspices of the U. S. Atomic Energy Commission. 


\section{DISCLAIMER}

This report was prepared as an account of work sponsored by an agency of the United States Government. Neither the United States Government nor any agency Thereof, nor any of their employees, makes any warranty, express or implied, or assumes any legal liability or responsibility for the accuracy, completeness, or usefulness of any information, apparatus, product, or process disclosed, or represents that its use would not infringe privately owned rights. Reference herein to any specific commercial product, process, or service by trade name, trademark, manufacturer, or otherwise does not necessarily constitute or imply its endorsement, recommendation, or favoring by the United States Government or any agency thereof. The views and opinions of authors expressed herein do not necessarily state or reflect those of the United States Government or any agency thereof. 


\section{DISCLAIMER}

Portions of this document may be illegible in electronic image products. Images are produced from the best available original document. 
Alternating Current Polishing and Electroetching of Metals

Aiternating current electropolishing and electroetching as a generally applicable technique in metallography was suggested by Reiswig's use of an AC method on Os-Ir alloys (1). The etching of plutonium has been a continuing problem, the previous techniques consisting mainly of DC electrolytic polishing and etching. The $A C$ technique was investigated because it seemed to offer an alternative to both DC electrolytic and chemical etching. After the success of the AC method on plutonium allotropes was achieved, a number of other metals were tried and, using only four electrolytes, a considerable degree of success was achieved.

Figure 1 is a schematic drawing of the equipment set-up.

The four electrolytes used are listed in Table $I$. The conditions for the AC etching of plutonium, but not the other metals, were extensively investigated with respect to variations in solutions, voltages, and current densities. The examples of other metals (figures 2-21) are given only to suggest the broad applicability of the AC technique and to indicate the results that may be obtained.

The conditions for specific metals are listed under the photomicrographs. The general results on metals are listed in Table II. Although a number of metals were etched poorly or not at all by the AC technique (see Table II), it is very likely that many of them and others could be successfully etched in solutions other then those listed in Table I. 


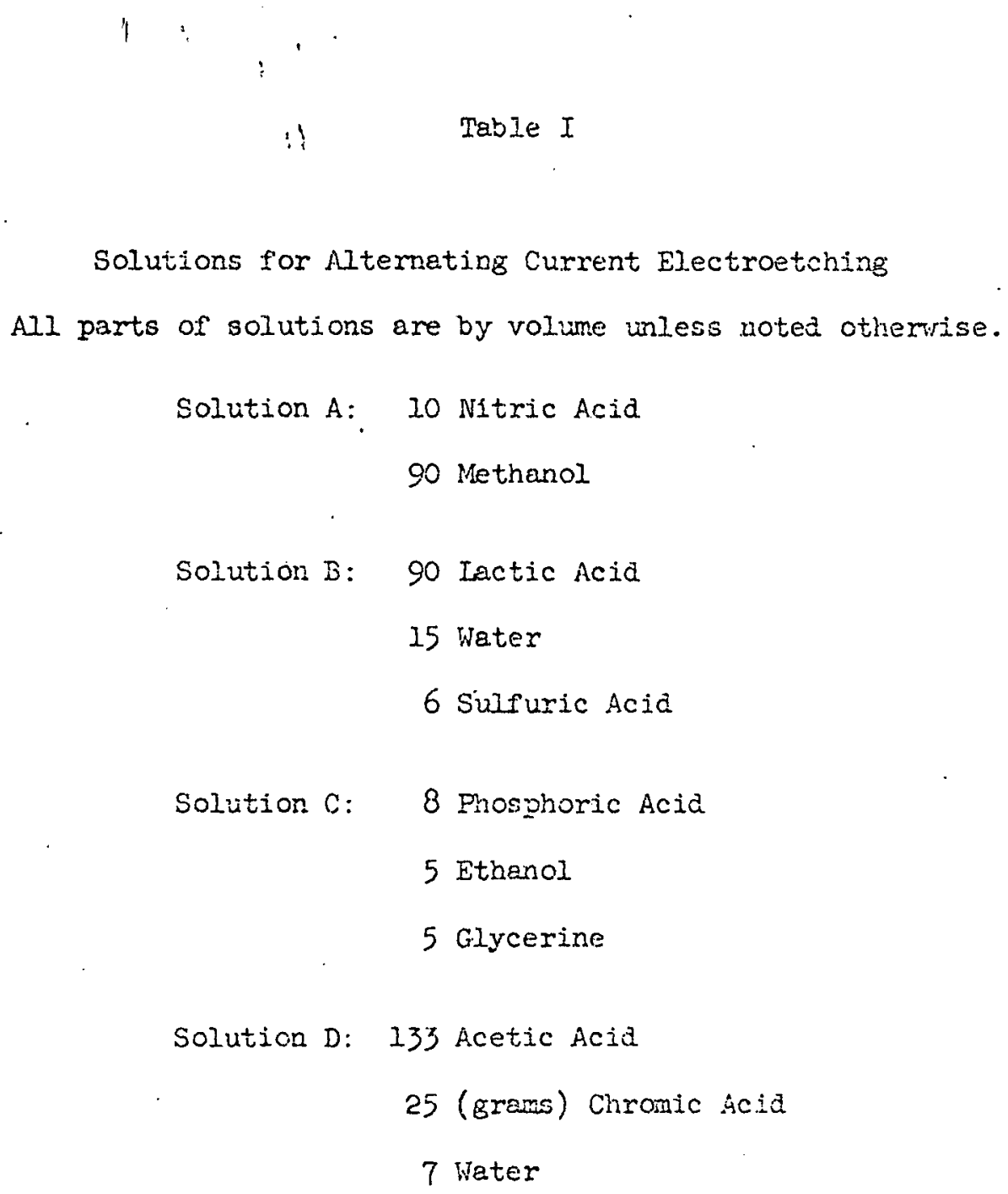


Table II

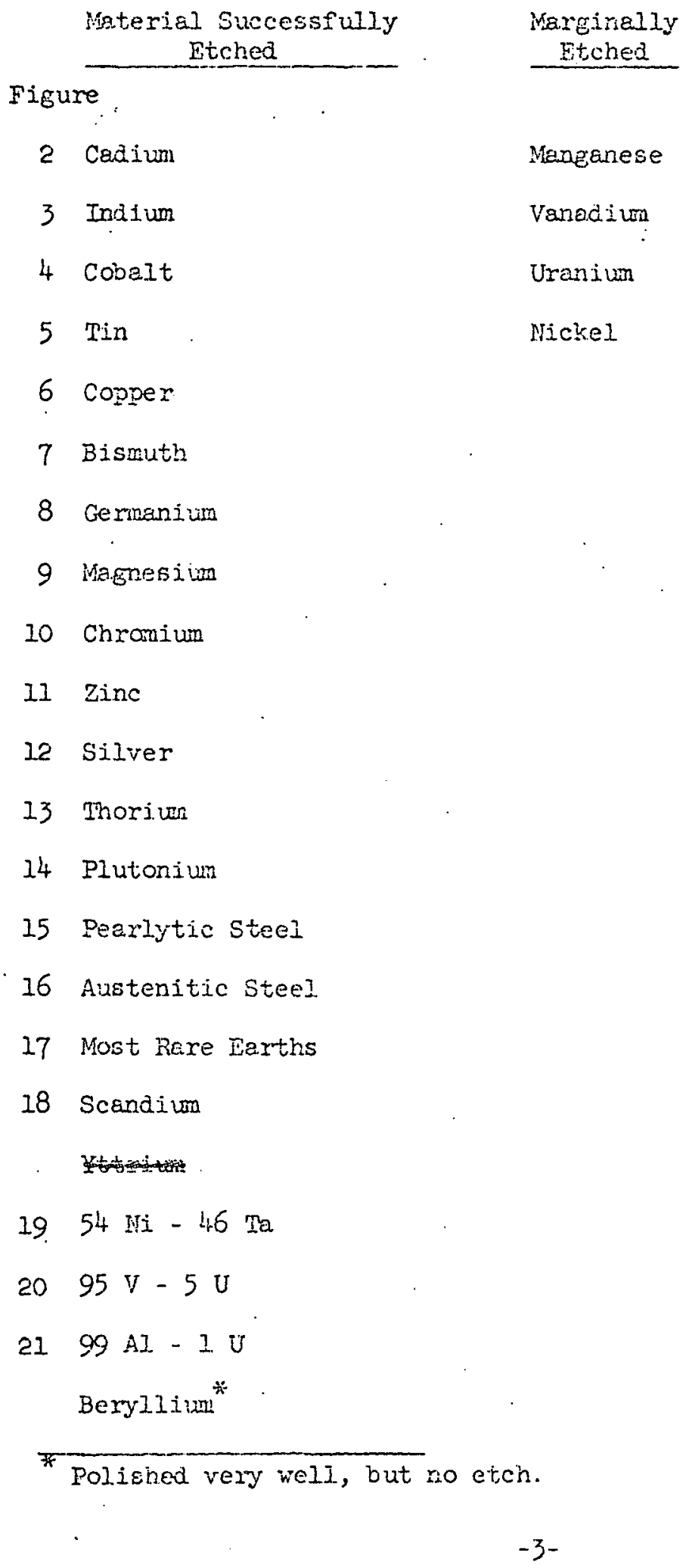




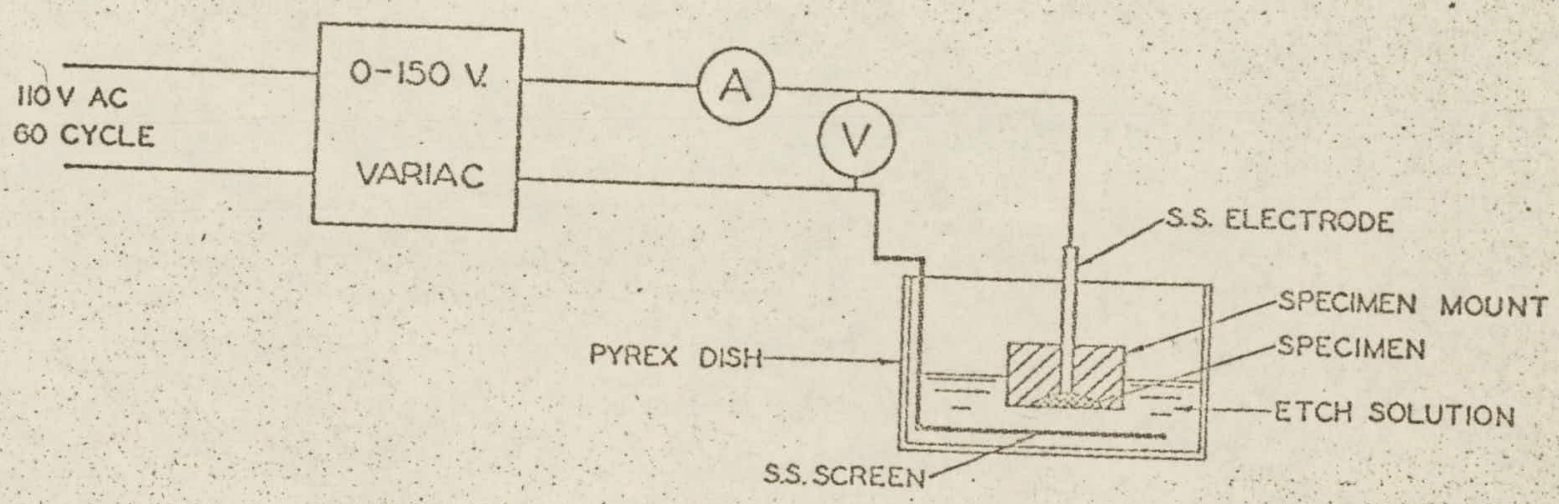

Figure I Schematic of AC etching equipment set-up. (The specimen probe and electrode are made of stainiess steel) 


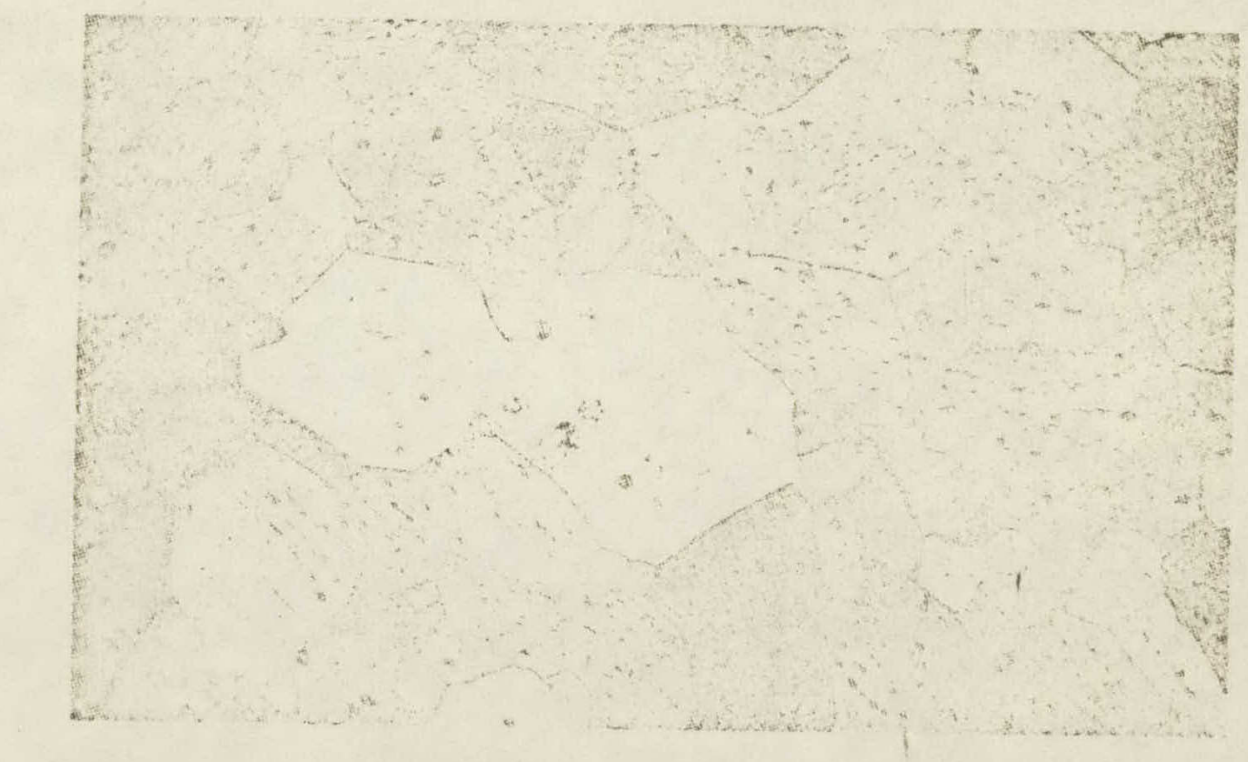

Figure 2

Cadiun 500x

Solution $B$

40 volts $-10 \mathrm{sec}-1 \mathrm{~cm} / \mathrm{sq} \mathrm{cm}$

Etch, polish altematively to remove cold work

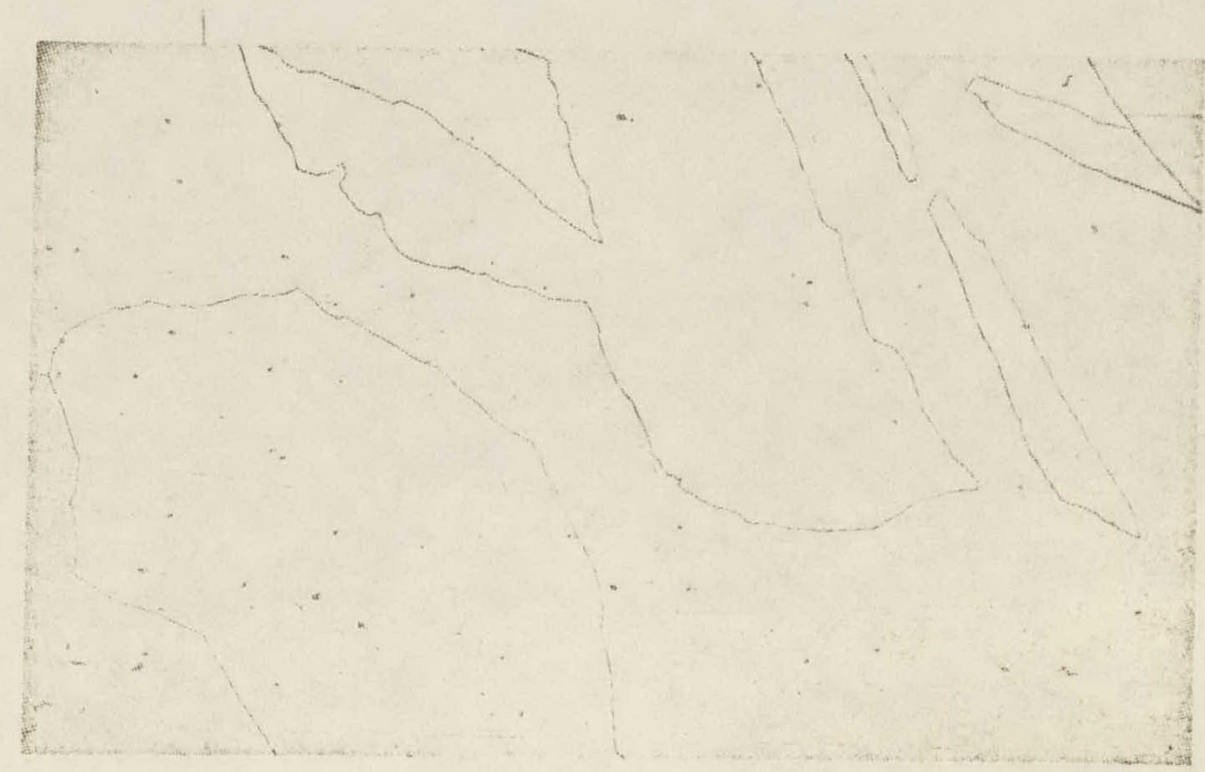

Figure 3

Indium $100 \mathrm{X}$

Solution $B$

30 volts - $10 \mathrm{sec}$

$.6 \mathrm{amp} / \mathrm{sq} \mathrm{cm}$ 


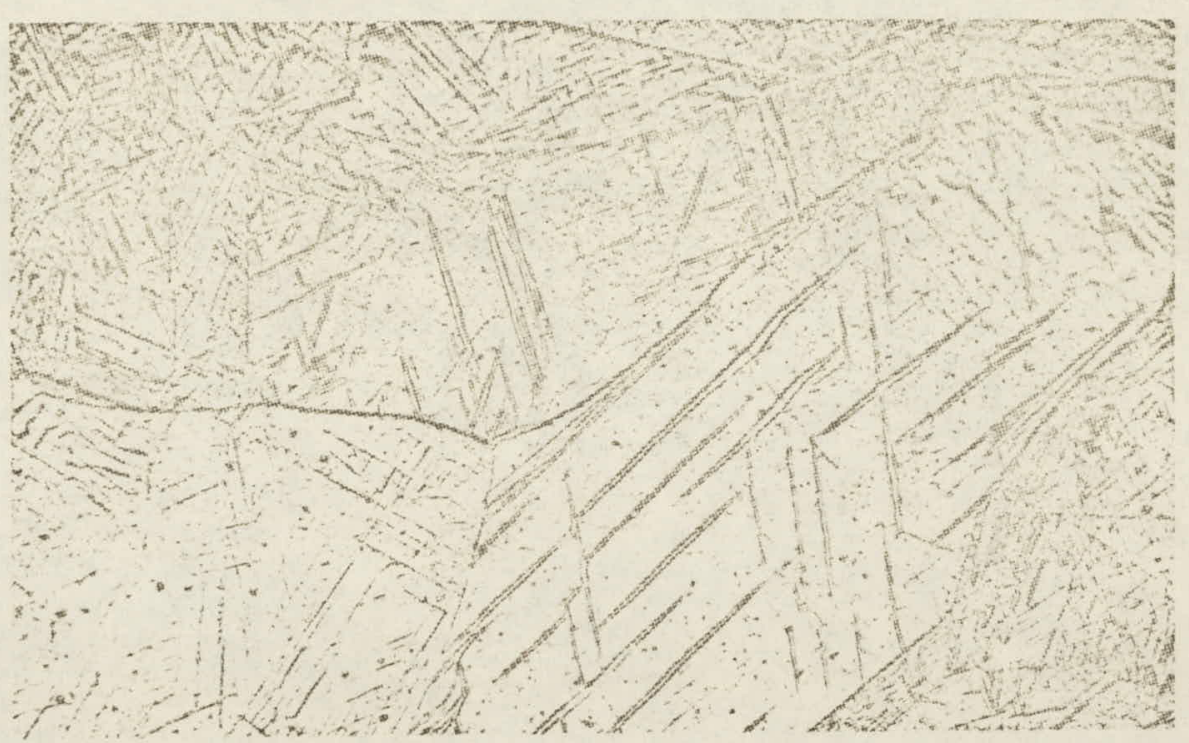

Figure 4

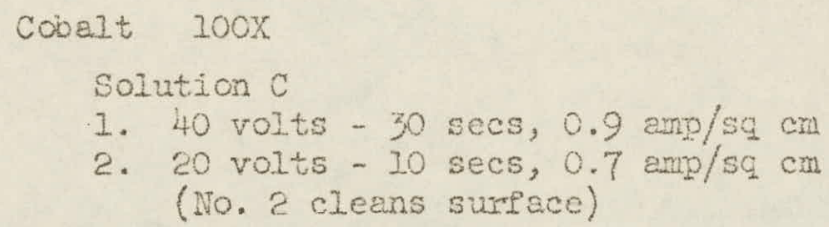
(No. 2 cleans surface)

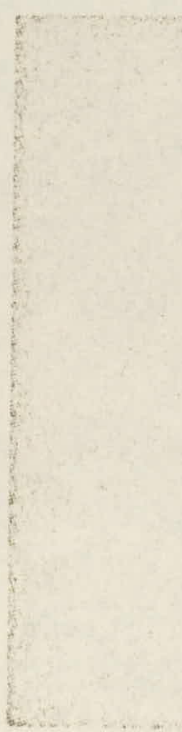

Figure 5

min $500 x$

Solution $B$

30 volts - 10 secs, $0.5 \mathrm{amp} / \mathrm{sq} \mathrm{cm}$

+ 10\% Mital dip. to clean up 


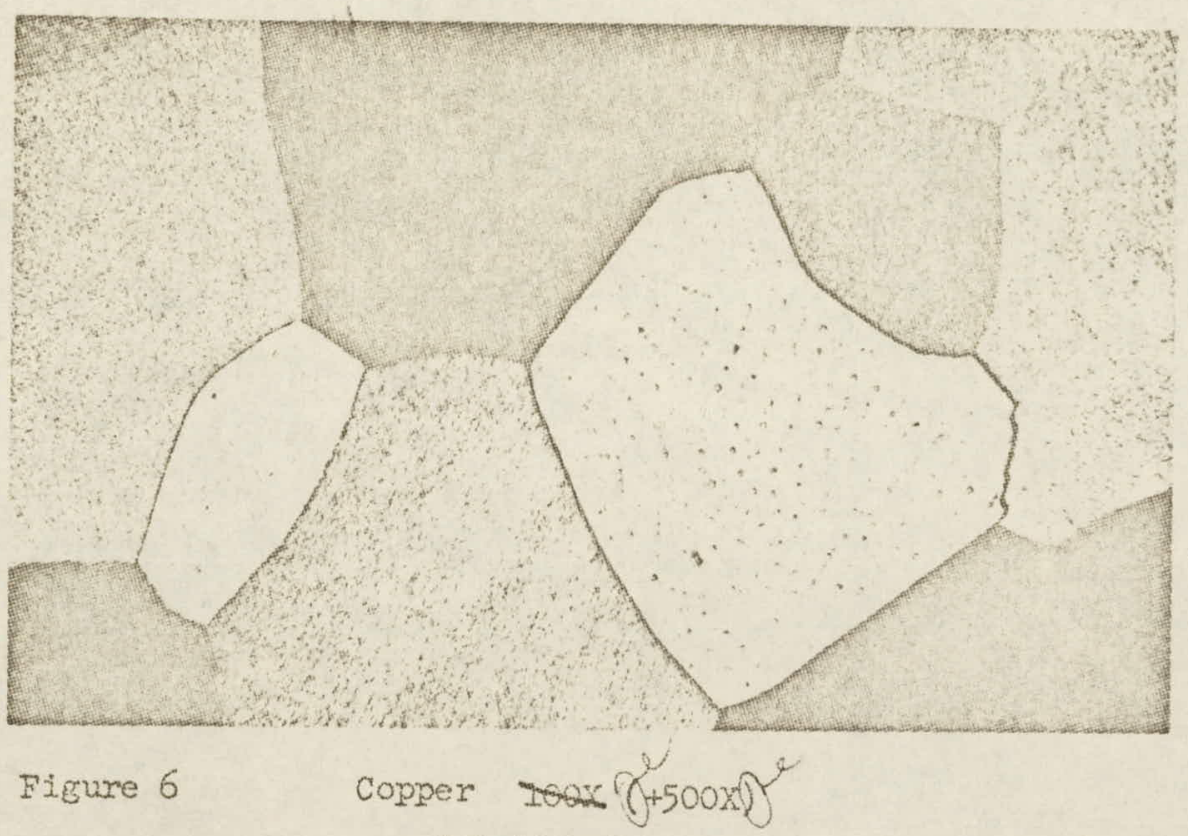

Solution $B$

40 volts - $35 \mathrm{sec}$

$2.1 \mathrm{amp} / \mathrm{sq} \mathrm{cm}$

Shorter time for smaller grain sizes

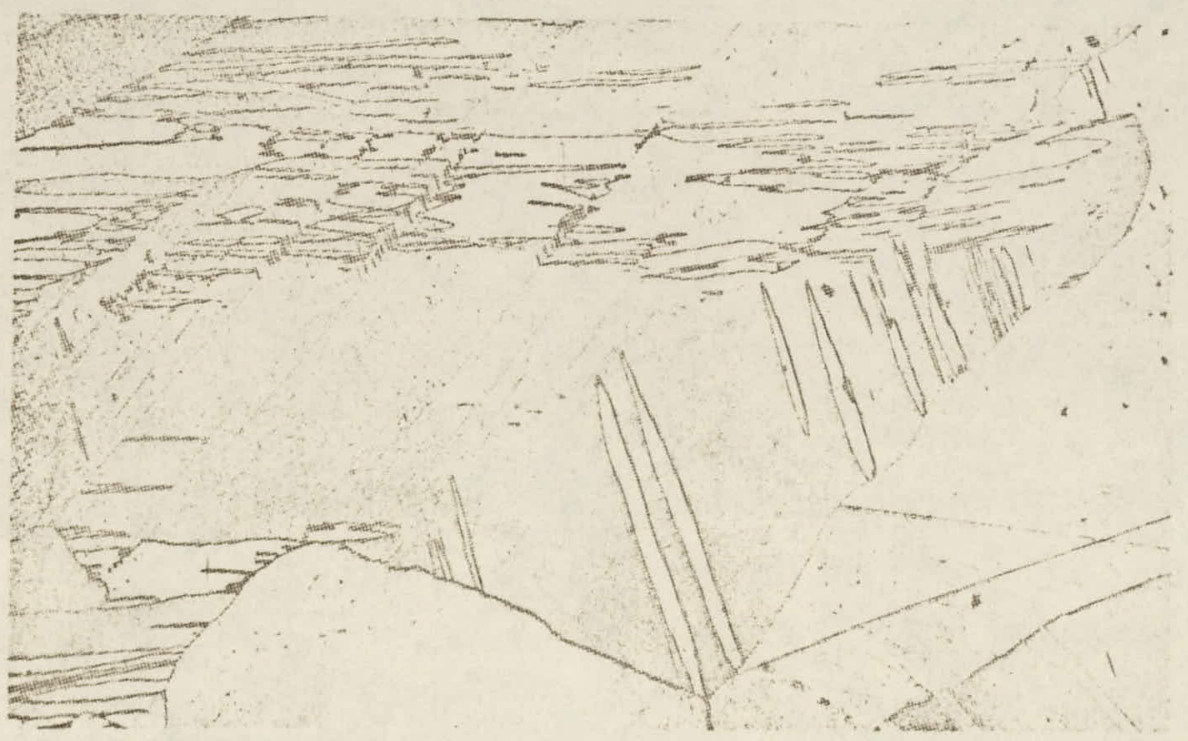

Figure 7

Bismuth $100 \mathrm{x}$

Solution A

30 volts - $3 \mathrm{sec}$

$10 \mathrm{amp} / \mathrm{sq} \mathrm{cm}$ 

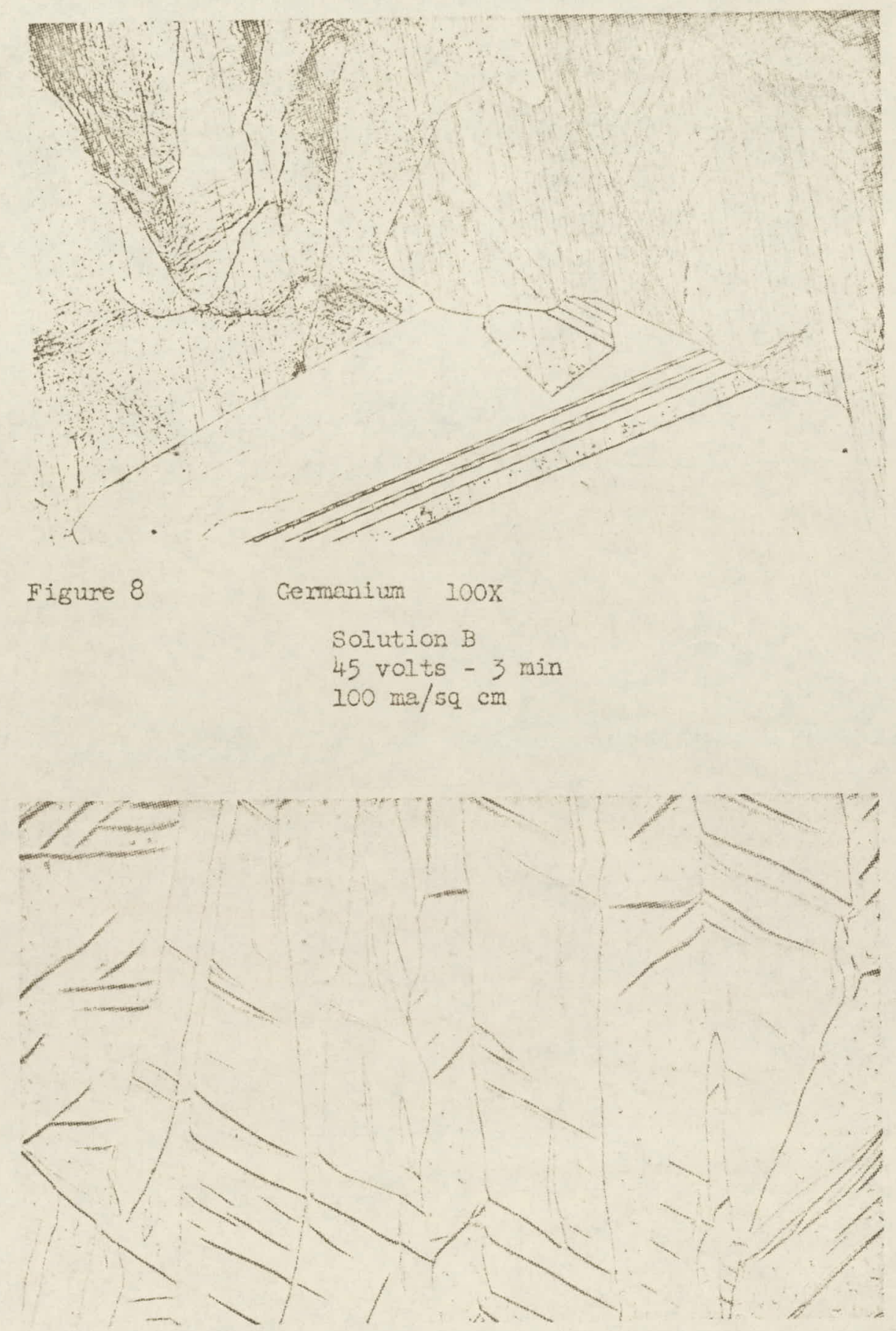

Figure 9

$$
\begin{aligned}
& \text { Magnesium } 500 \mathrm{X}(+100 \mathrm{X}) \\
& \text { Solution } \mathrm{A} \\
& 20 \text { volts }-10 \mathrm{sec}
\end{aligned}
$$




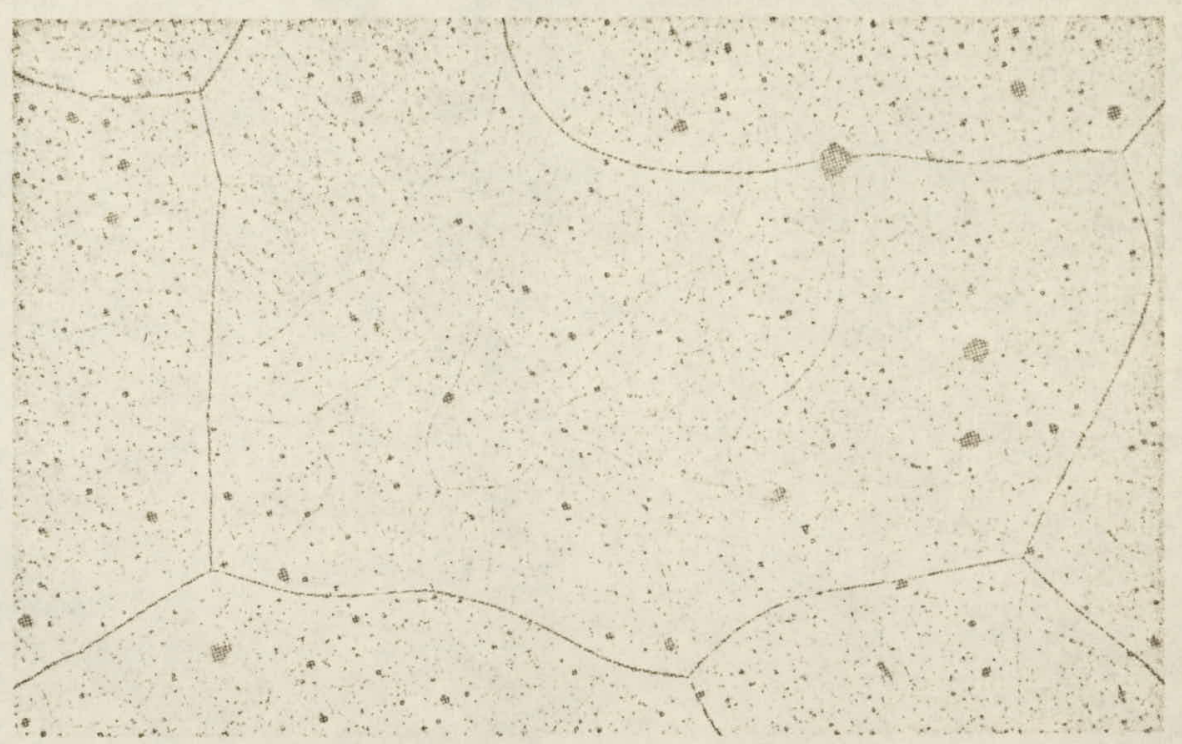

Figure 10

Chromium $200 x$

Solution $30 \% \mathrm{Nital}$ ( $\mathrm{Cr}$ doped)

10 volts - $5 \mathrm{sec}$

$4.0 \mathrm{amps} / \mathrm{sq} \mathrm{cm}$
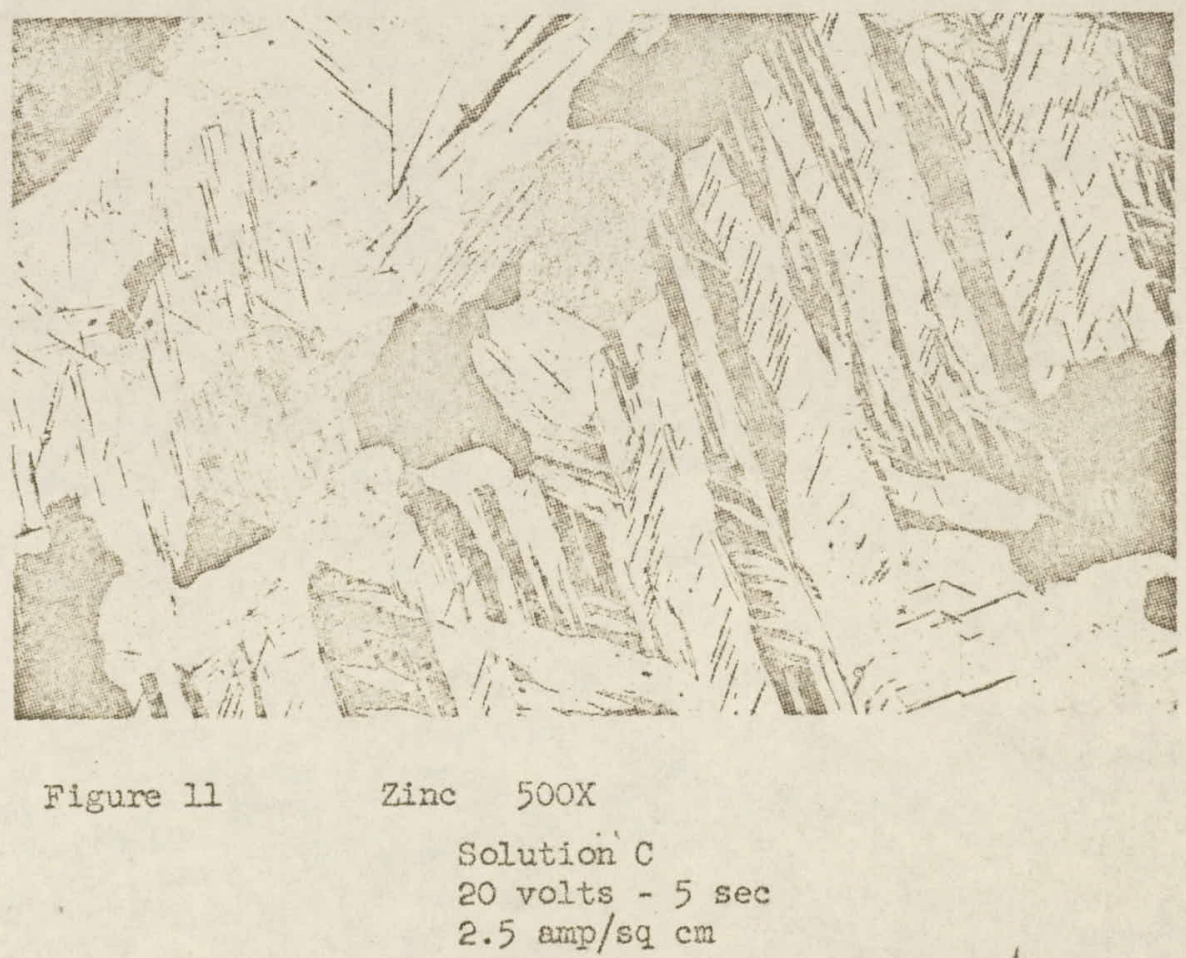

Figure 11

Zine $500 \mathrm{x}$

Solution $\mathrm{C}$

20 volts - $5 \mathrm{sec}$

$2.5 \mathrm{amp} / \mathrm{sq} \mathrm{cm}$ 


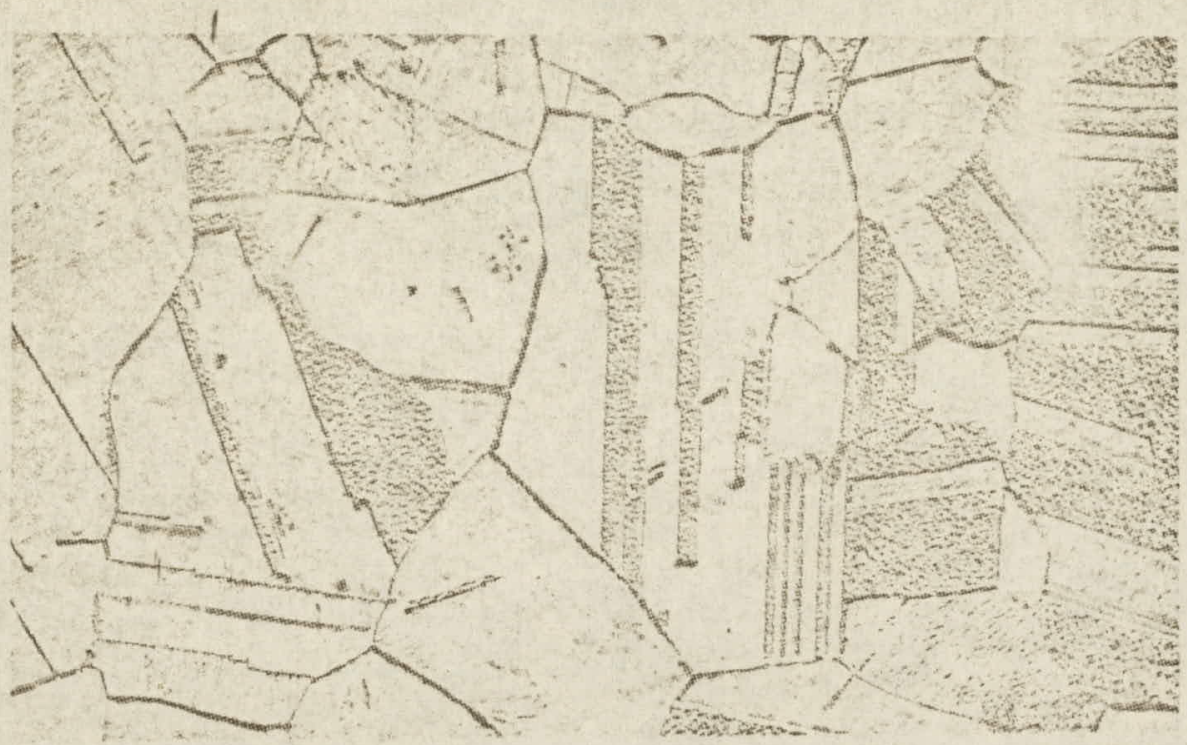

Figure 12

Silver 500x

Solution C

20 volts - $10 \mathrm{sec}$

$2.8 \mathrm{amp} / \mathrm{sq} \mathrm{cm}$

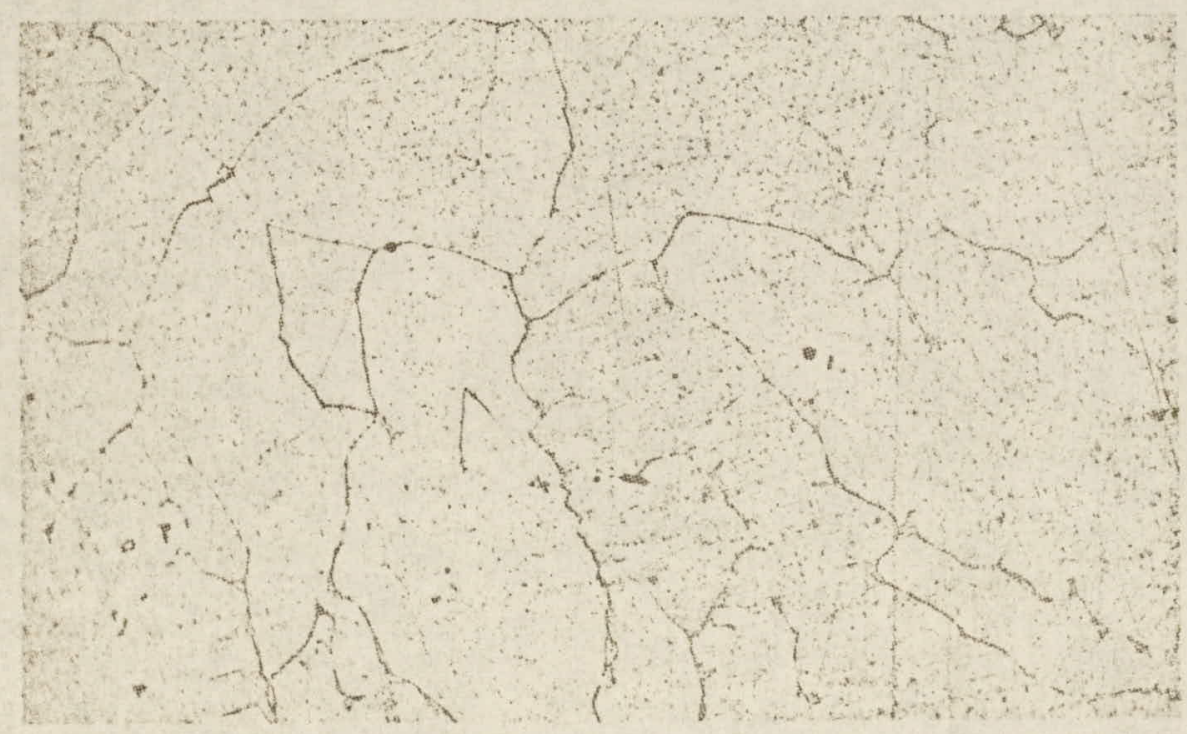

Figure 13

Thorium 100x

Solution $D$

50 volts $-35 \mathrm{sec}$

$0.4 \mathrm{amp} / \mathrm{sq} \mathrm{cm}$ 


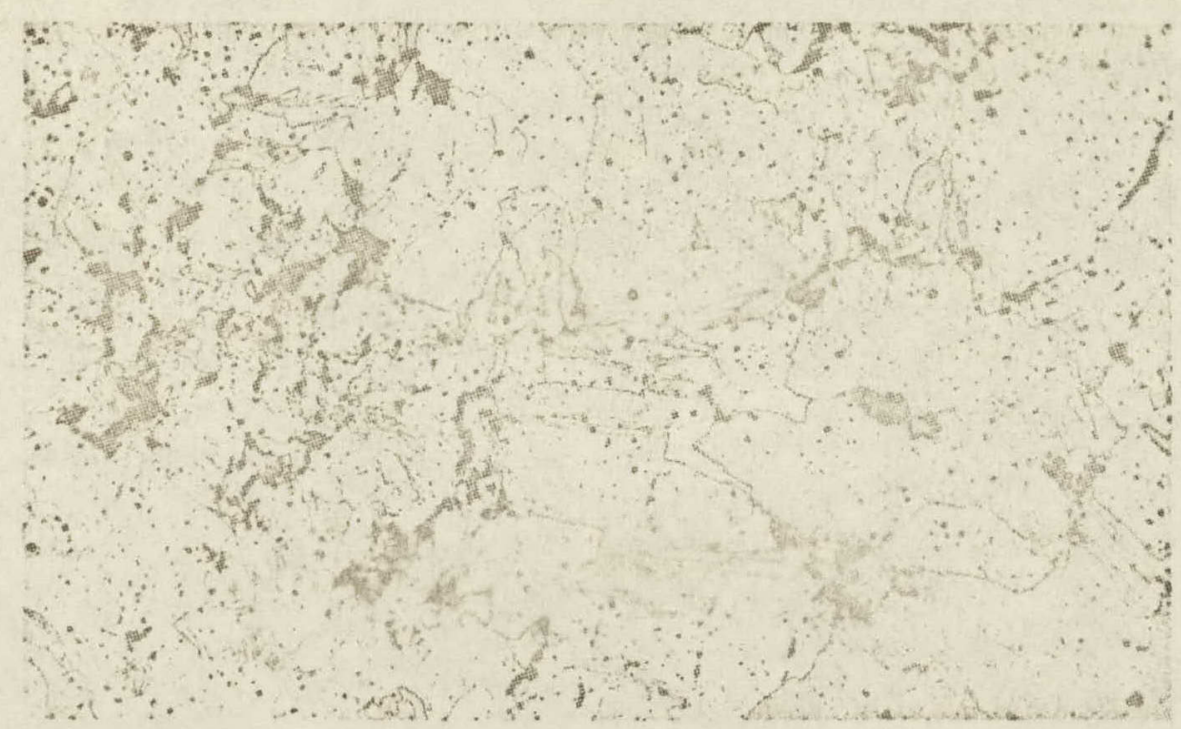

Figure 14

$$
\begin{aligned}
& \text { Alpha plutonium } 100 \mathrm{X} \\
& \text { Solution A } \\
& 5 \mathrm{volts}-20 \mathrm{sec} \\
& .8 \mathrm{~mm} / \mathrm{sq} \mathrm{cm}
\end{aligned}
$$

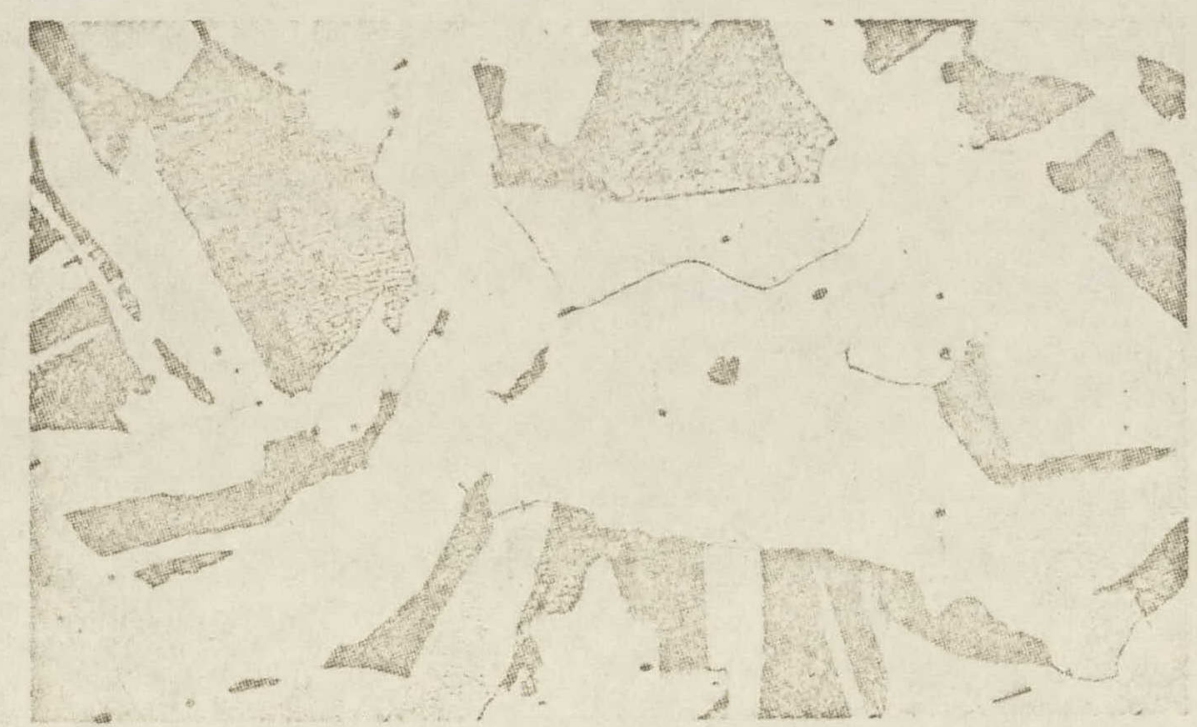

Figure 15

$$
\begin{aligned}
& \text { Mo20 Steel - Pearlite + Perite } 500 \mathrm{X} \\
& \text { Solution B } \\
& 20 \mathrm{volts}-10 \mathrm{sec} \\
& 1 \mathrm{amp} / \mathrm{sq} \mathrm{cm}
\end{aligned}
$$




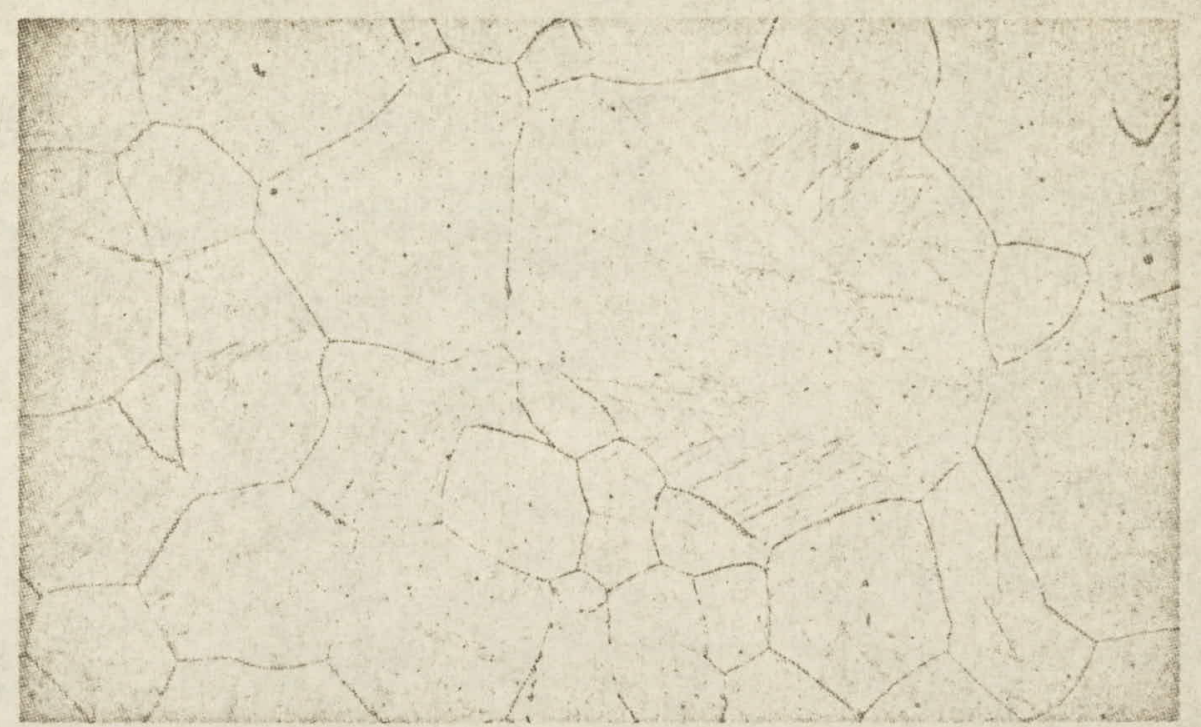

Figure 16

Austenite + martensite needles $100 \mathrm{X}(+1000 \mathrm{X})$

Solution $B$

20 volts - $10 \mathrm{sec}$

$0.6 \mathrm{amp} / \mathrm{sq} \mathrm{cm}$

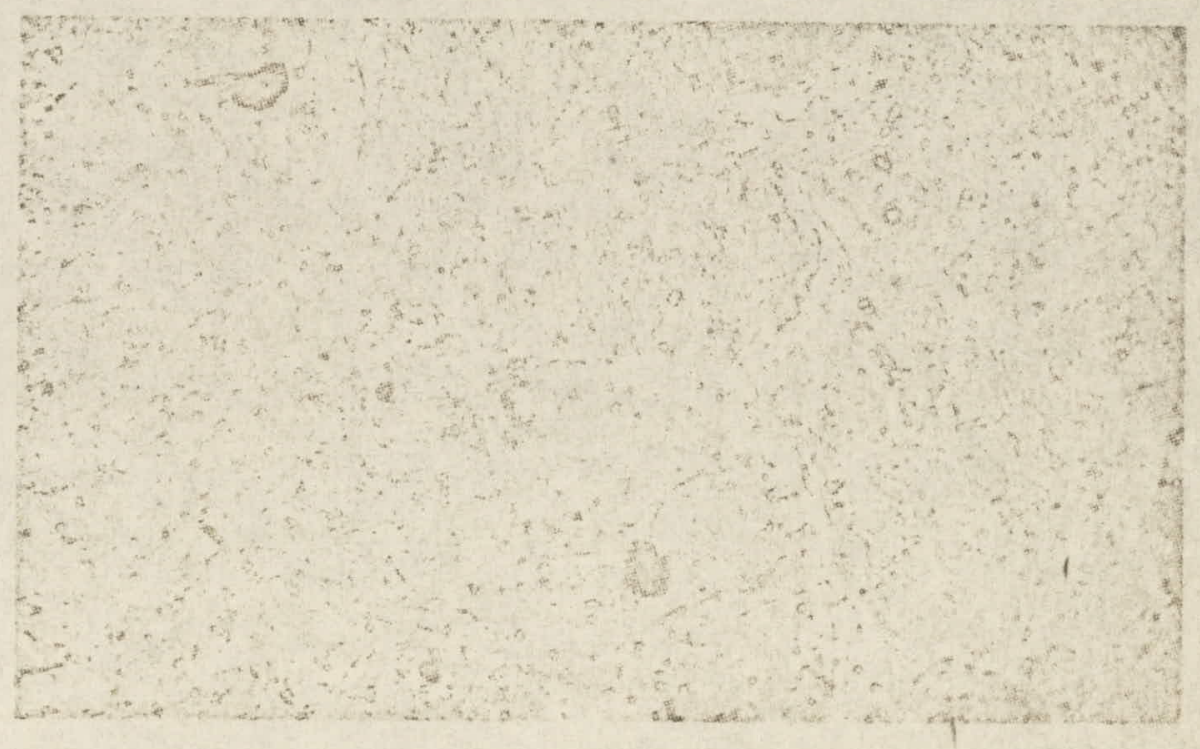

Figure 17

Samarium $200 X$

Solution $B$

10 volts - $10 \mathrm{sec}$

$70 \mathrm{ma} / \mathrm{sq} \mathrm{cm}$ 


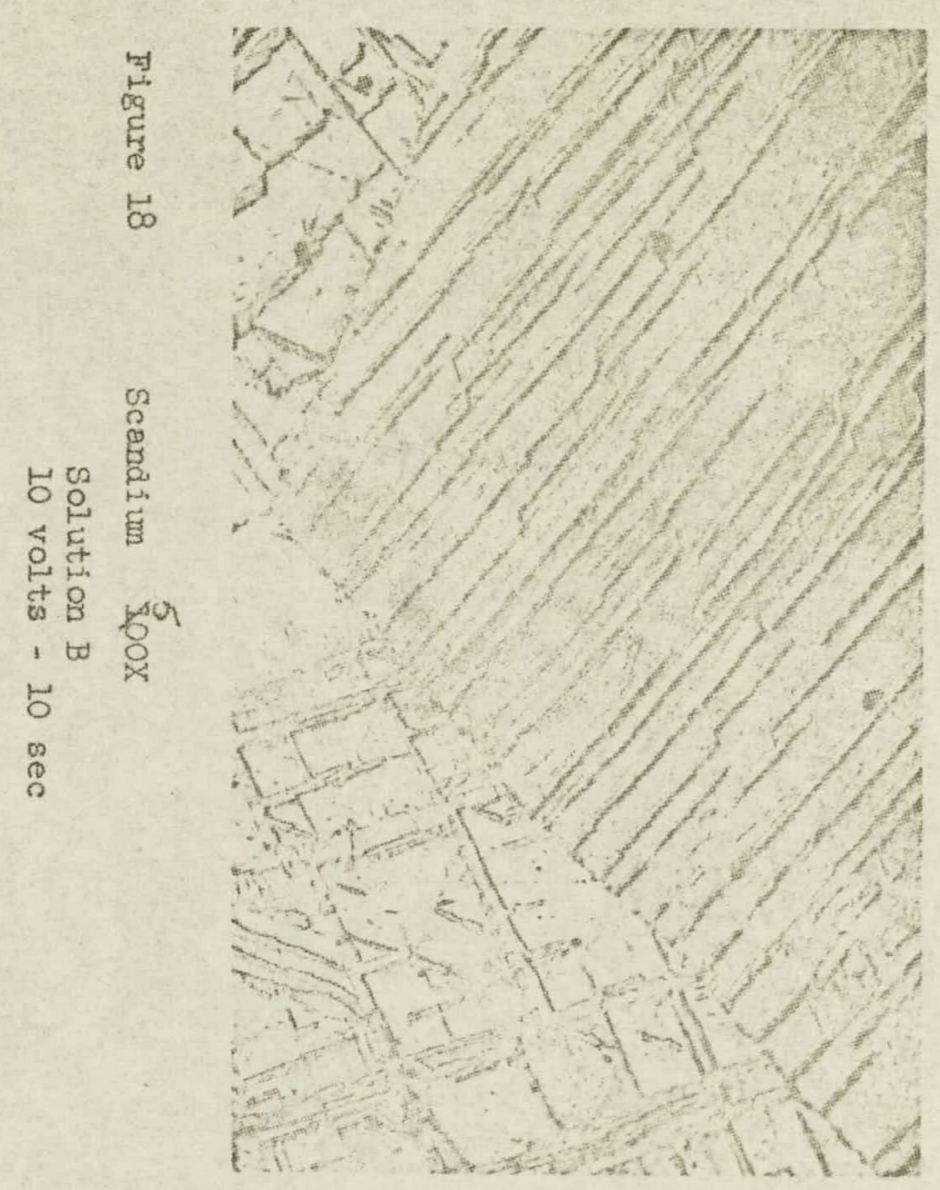



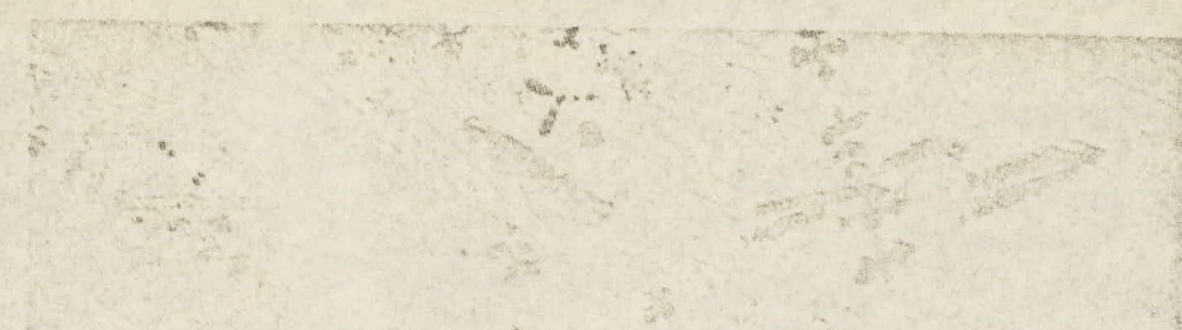

$$
\text { है: }
$$$$
i^{2}
$$

Figure 19

$54 \mathrm{NI}-46 \mathrm{Ie}$ Three phases $500 \mathrm{X}$

Solution $C$

40 volts - $10 \mathrm{sec}$

$2 \operatorname{arap} / \mathrm{sq} \mathrm{cm}$

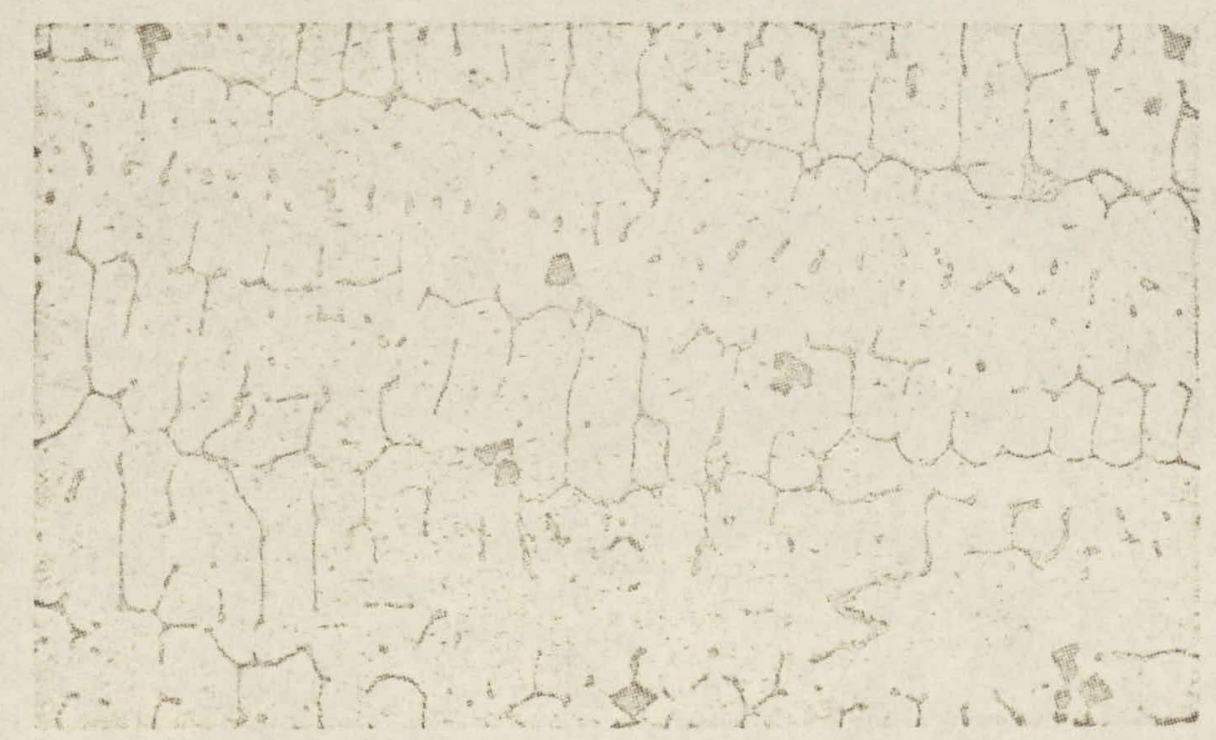

Figure 20

$$
\begin{aligned}
& 95 \mathrm{~V}-5 \mathrm{U} \quad 100 \mathrm{X} \\
& \text { Solution B } \\
& 20 \mathrm{volts}-10 \mathrm{sec} \\
& 1 \mathrm{amp} / \mathrm{sq} \mathrm{cm}
\end{aligned}
$$




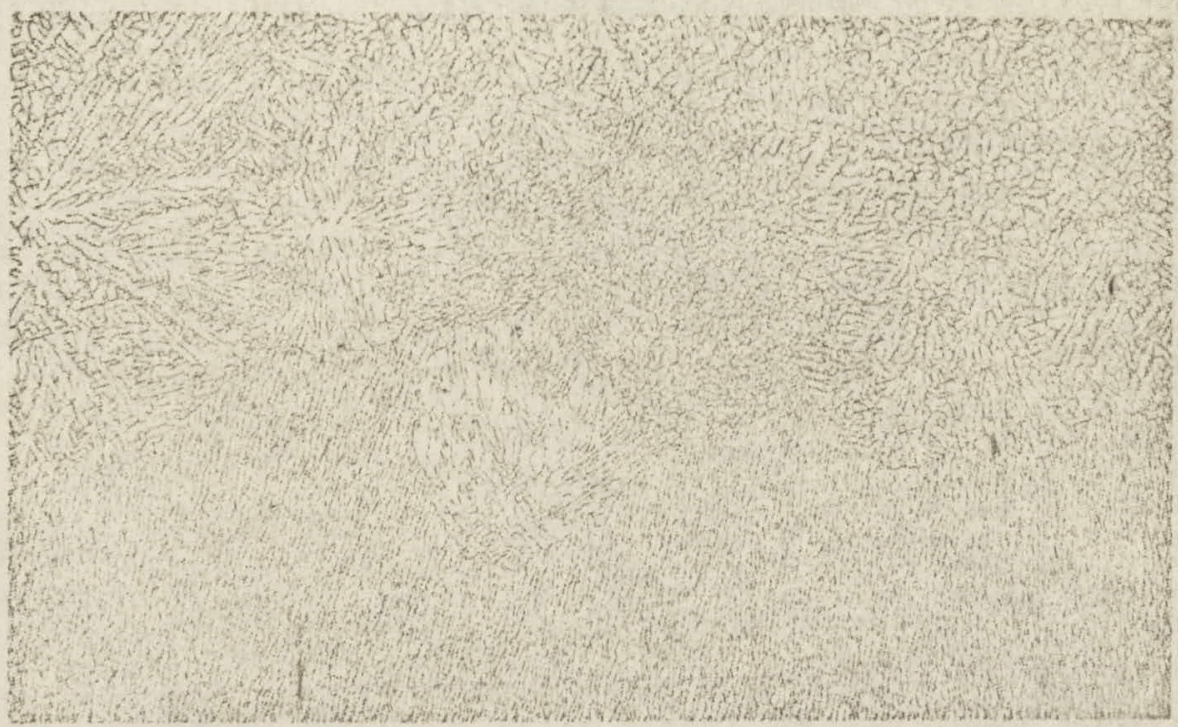

Figure 21 $99 \mathrm{Al}-1 \mathrm{U} 100 \mathrm{X}$

Solution C
$40 \mathrm{volts}-10 \mathrm{sec}$
$1 \mathrm{amg} / \mathrm{sq} \mathrm{cm}$


1. Schematic of AC etching equioment set-up. (The specimen probe and electrode are made of stainless steel.)

2. Cedium $500 \mathrm{x}$, Solution 8,40 volts $-10 \mathrm{sec}-1 \mathrm{am} / \mathrm{sq} \mathrm{cm}$, Etch, polish altematively to remove cold work.

3. Indium 100K, Solution $B$, 30 volts $-10 \mathrm{sec}, .6 \mathrm{amp} / \mathrm{sq} \mathrm{cn}$.

4. Cobalt 1100X, Solution C, 1.) $40 \mathrm{volis}-30$ secs $-0.9 \mathrm{arm} / \mathrm{sq} \mathrm{cm}$, 2.) 20 volts - 10 secs $-0.7 \mathrm{amp} / \mathrm{sq} \mathrm{cm}$, (iro. 2 cleans slirface.

5. Tin 500X, soiution B, 30 volts $-10 \mathrm{secs}, 0.5 \mathrm{erp} / \mathrm{sq} \mathrm{cm}$, + $10 \mathrm{Nital}$ aip. to clean up.

6. Copper $100 \times(+500 \mathrm{X})$, Solution $B, 40$ volts $-35 \mathrm{sec}, 2.1$ aimp/sq cm, Shorter time for smaller grein sizes.

7. Bismuth 100X, Solution A, 30 volts $-3 \mathrm{sec}, 10 \mathrm{emp} / \mathrm{sq} \mathrm{cm}$.

8. Germanium 100X, Solution B, 45 volts $-3 \mathrm{~min}, 100 \mathrm{ma} / \mathrm{sq} \mathrm{cm}$.

9. Magnesium $500 \mathrm{x}(+100 \mathrm{x})$, Solution $\mathrm{A}, 20$ volts $-10 \mathrm{sec}$.

10. Chromium 100X, 'Solution $30 \% \mathrm{Nital}$ (Cr dopea), 20 volts $-5 \mathrm{sec}$, $4.0 \mathrm{amps} / \mathrm{sq} \mathrm{cm}$.

11. Zinc 500x, Solution $\mathrm{C}, 20$ rolts $-5 \mathrm{sec}, 2.5 \mathrm{amp} / \mathrm{sq} \mathrm{cm}$.

12. Silver 500X, Solution C, 20 volts $-10 \mathrm{sec}, 2.8 \mathrm{enp} / \mathrm{se} \mathrm{cm}$.

13. Thorium 100X, Solution D, 50 volts $-35 \mathrm{sec}, 0.4 \mathrm{smp} / \mathrm{sq} \mathrm{cm}$.

14. Alphe plutonium 100X, solution $\mathrm{A}, 5 \mathrm{volts}-20 \mathrm{sec}, .8 \mathrm{smp} / \mathrm{sq} \mathrm{cm}$.

15. Mozo Steel - Pearlite + ferite 500X, Solution B, 20 volts - $10 \mathrm{sec}$, I emp/sq cm.

16. Austenite + maxtensite needles $100 \mathrm{X}(+1000 \mathrm{X})$, solution $B, 20$ volts $10 \mathrm{sec}, 0.6 \mathrm{mp} / \mathrm{sq} \mathrm{cm}$.

17. Samarium 100X, Solution $B, 10$ volts $-10 \mathrm{sec}, 70 \mathrm{ma} / \mathrm{sq} \mathrm{cm}$.

18. Scanaium 100X, solution $B$, 10 volts - $10 \mathrm{sec}$,

19. $54 \mathrm{NI}$ - $46 \mathrm{ma}$ inree phases 500x, Solution C, 40 volts - $10 \mathrm{sec}$, $2 \operatorname{amp} / \mathrm{sq} \mathrm{cm}$.

20. $95 \mathrm{~V}-5 \mathrm{U}$ 100X, Solution $\mathrm{B}, 20 \mathrm{volts}-10 \mathrm{sec}, 1 \mathrm{amig} / \mathrm{sq} \mathrm{cm}$.

21. $99 \mathrm{Al}-\mathrm{I} \mathrm{U}$ 100X, Solution $\mathrm{C}, 40 \mathrm{volts}-10 \mathrm{sec}, 1 \mathrm{amp} / \mathrm{sg} \mathrm{cm}$. 


\section{RETERENCE}

1. R. D. Reiswig and J. M. Dickinson, "The Osmium-Iriaium Equilibrium Diagran", Mrans. Hetallurgical Society AINe, 230, pp. 469-472, 19034. 